\title{
Protein Translocation into the
} Intermembrane Space and Matrix of Mitochondria: Mechanisms and Driving Forces

\author{
Sandra Backes and Johannes M. Herrmann* \\ Cell Biology, University of Kaiserslautern, Kaiserslautern, Germany
}

Mitochondria contain two aqueous subcompartments, the matrix and the intermembrane space (IMS). The matrix is enclosed by both the inner and outer mitochondrial membranes, whilst the IMS is sandwiched between the two. Proteins of the matrix are synthesized in the cytosol as preproteins, which contain amino-terminal matrix targeting sequences that mediate their translocation through translocases embedded in the outer and inner membrane. For these proteins, the translocation reaction is driven by the import motor which is part of the inner membrane translocase. The import motor employs matrix Hsp70 molecules and ATP hydrolysis to ratchet proteins into the mitochondrial matrix.

OPEN ACCESS

Edited by:

Ophry Pines,

Hebrew University of Jerusalem, Israel

Reviewed by:

Abdussalam Azem,

Tel Aviv University, Israel

Agnieszka Chacinska,

International Institute of Molecular and

Cell Biology, Poland

*Correspondence:

Johannes M. Herrmann

hannes.herrmann@biologie.uni-kl.de

Specialty section:

This article was submitted to Protein Folding, Misfolding and

Degradation,

a section of the journal

Frontiers in Molecular Biosciences

Received: 20 October 2017 Accepted: 24 November 2017 Published: 07 December 2017

Citation:

Backes S and Herrmann JM (2017)

Protein Translocation into the

Intermembrane Space and Matrix of

Mitochondria: Mechanisms and

Driving Forces.

Front. Mol. Biosci. 4:83.

doi: 10.3389/fmolb.2017.00083
Most IMS proteins lack presequences and instead utilize the IMS receptor Mia40, which facilitates their translocation across the outer membrane in a reaction that is coupled to the formation of disulfide bonds within the protein. This process requires neither ATP nor the mitochondrial membrane potential. Mia40 fulfills two roles: First, it acts as a holdase, which is crucial in the import of IMS proteins and second, it functions as a foldase, introducing disulfide bonds into newly imported proteins, which induces and stabilizes their natively folded state. For several Mia40 substrates, oxidative folding is an essential prerequisite for their assembly into oligomeric complexes. Interestingly, recent studies have shown that the two functions of Mia40 can be experimentally separated from each other by the use of specific mutants, hence providing a powerful new way to dissect the different physiological roles of Mia40. In this review we summarize the current knowledge relating to the mitochondrial matrix-targeting and the IMS-targeting/Mia40 pathway. Moreover, we discuss the mechanistic properties by which the mitochondrial import motor on the one hand and Mia40 on the other, drive the translocation of their substrates into the organelle. We propose that the lateral diffusion of Mia40 in the inner membrane and the oxidation-mediated folding of incoming polypeptides supports IMS import.

Keywords: brownian ratchet, disulfide bond, foldase, holdase, Mia40, mitochondria, protein import

\section{INTRODUCTION}

Mitochondria are essential organelles of eukaryotic cells that carry out a large range of different activities (Figure 1). Only a very small proportion of the $\sim 1,000$ mitochondrial proteins are synthesized by mitochondrial ribosomes ( 8 in baker's yeast, 13 in humans, and 67 in Reclinomonas americana, the organism with the most complex mitochondrial genome; Lang et al., 1997). All 


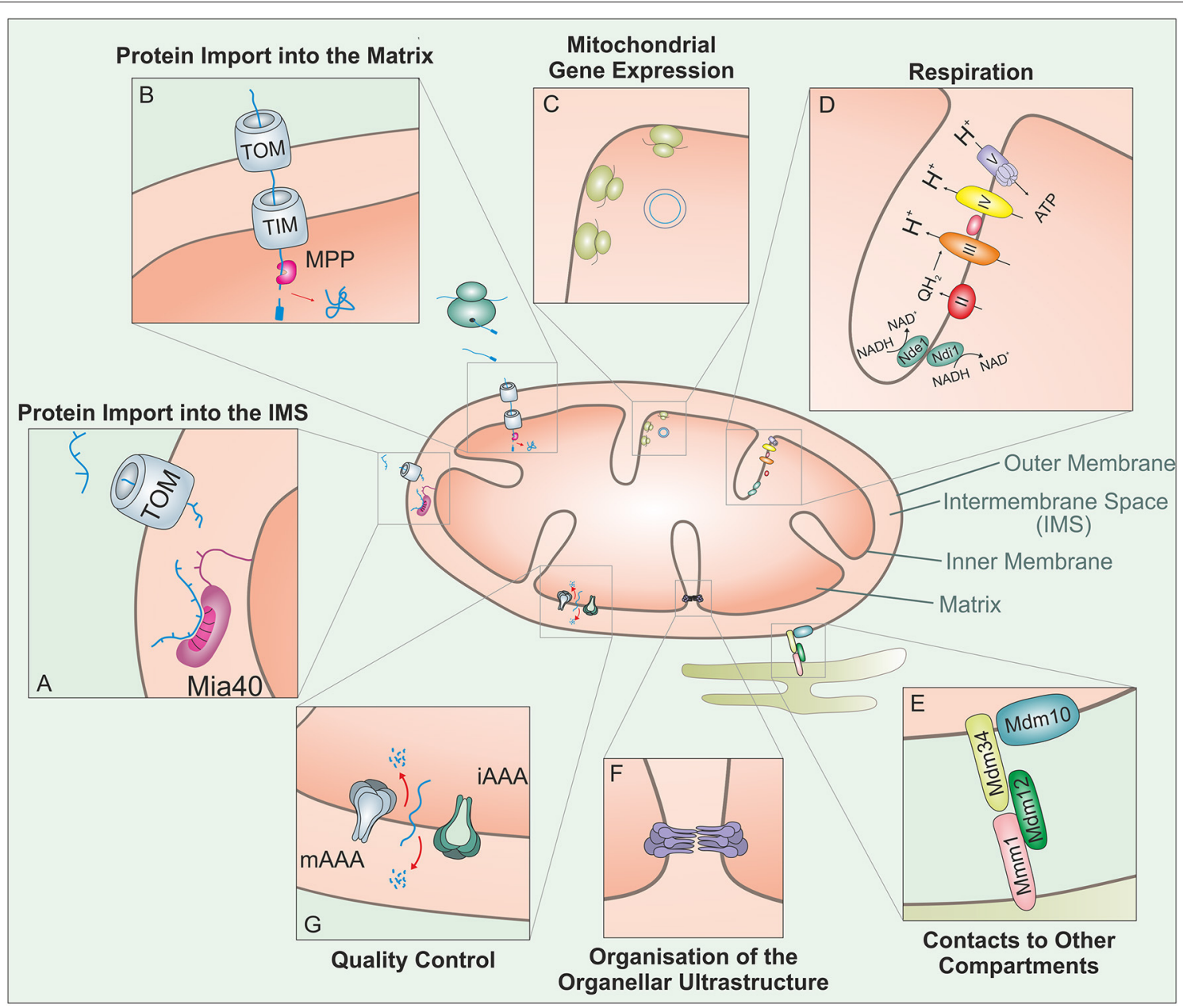

FIGURE 1 | Mitochondria carry out a large variety of different biological activities. This figure shows some of these functions which are relevant in the context of this review: The vast amount of mitochondrial proteins need to be imported from the cytosol. (A) Proteins targeted to the IMS enter through the TOM complex and bind to the IMS receptor Mia40, which is responsible for the introduction of disulfide bonds and concomitant protein folding. (B) Proteins with an N-terminal MTS are guided to the matrix through the TOM and TIM complex. The mitochondrial processing peptidase (MPP) removes the MTSs from preproteins before they can fold into their native structures. (C) Mitochondria contain a complete genetic system for replication, transcription and translation that is entirely distinct from that in the nucleus/cytosol. The mitochondrial translation system is membrane-bound and specialized on the synthesis of a small number of very hydrophobic polypeptides. (D) Mitochondria contain a large number of metabolic enzymes that carry out a variety of catabolic and anabolic pathways. Of particular abundance and relevance are the complexes of the respiratory chain, which use the transfer of electrons to generate an electrochemical gradient which drives the synthesis of the vast majority of the cellular ATP. (E) Mitochondria interact with many other cellular compartments. The ER-mitochondria encounter structure (ERMES) tethers mitochondria to the ER, presumably to facilitate the exchange of calcium and phospholipids between their membranes. (F) The ultrastructure of mitochondria depends on the function of a number of protein complexes. Of particular importance is the "cristae organizing system" (MICOS) which participates in the formation of cristae junctions and contact sites of the inner and outer membrane. (G) Mitochondrial peptidases can regulate different mitochondrial functions through proteolytic processing and protein degradation. In addition to a number of soluble proteases, the inner membrane contains two very important ATP-hydrolysing protease complexes that belong to the AAA (ATPases associated with different cellular activities) protein family: these i-AAA and m-AAA proteases expose their proteolytic domains to the IMS and membrane sides of the inner membrane, respectively.

other proteins are synthesized in the cytosol from where they are imported into mitochondria by a translocase in the outer membrane of mitochondria (TOM complex) and translocases in the inner membrane of mitochondria (TIM complexes). Proteins of the matrix and the inner membrane, each making up about $40 \%$ of all mitochondrial proteins (Calvo et al., 2016; Morgenstern et al., 2017), employ both TOM and TIM translocases and predominantly use similar mechanisms for their import process. These proteins include many of the enzymes that catalyze the major biochemical functions of the organelle in metabolic conversions and respiration, in the biosynthesis of lipids, iron-sulfur clusters, heme, and amino acids, or in the expression of mitochondrial genes (Figure 1). Proteins of the outer membrane and the IMS, each making up about $10 \%$ of the mitochondrial proteome, use a diversity of strategies to make their way into mitochondria. Outer membrane and IMS proteins play crucial roles in the communication with the cytosol and with other mitochondrial compartments, in the uptake of metabolites, 
lipids, or metal ions as well as with the regulation and execution of apoptosis (Wang and Youle, 2009; Herrmann and Riemer, 2010; Aaltonen et al., 2016; Miyata et al., 2016).

In this review we focus on the import of proteins into the IMS and discuss recent studies on the mechanisms of the Mia40mediated import reaction and compare this process to the wellstudied preprotein pathway which drives the translocation of matrix and inner membrane proteins.

\section{PROTEIN IMPORT INTO THE MITOCHONDRIAL MATRIX}

Proteins destined to the mitochondrial matrix are synthesized with amino terminal presequences that serve as matrix-targeting sequences (MTSs). These sequences form amphipathic helices that vary largely in primary sequence but are characterized by a length of about 15-60 residues, a net charge of +3 to +6 , the absence of negatively charged residues and a high content of hydroxylated amino acids (Vögtle et al., 2009; Calvo et al., 2017). MTSs are recognized by the receptors Tom 20/Tom 22 and Tom70 on the mitochondrial surface, which have considerable overlap in specificity and functionality (Brix et al., 1999; Fan et al., 2011). The binding of MTSs to the TOM receptors, particularly to Tom70, is facilitated by cytosolic chaperones of the Hsp70 and Hsp90 classes as well as by co-chaperones such as Stil (Young et al., 2003; Hansen et al., 2016; Hoseini et al., 2016).

Following receptor binding the preproteins are threaded through the protein-conducting channel of the TOM complex that is formed by the beta barrel protein Tom40 (Shiota et al., 2015) and are subsequently transferred to the TIM23 complex of the inner membrane. This translocase comprises three essential inner membrane proteins: Tim50, Tim23, and Tim17 (Figure 2). Tim50 exposes a large domain into the IMS that promotes the transfer of preproteins from the TOM to the TIM23 translocase and regulates the gating of the TIM channel (Mokranjac et al., 2009; Schulz et al., 2011; Bajaj et al., 2014). Tim23 and Tim17 are two structurally related multi-spanning inner membrane proteins that form the protein-conducting channel of the inner membrane (Truscott et al., 2001; Meier et al., 2005; Zarsky and Dolezal, 2016). Tim17, which shows the highest degree of sequence conservation of all TOM and TIM subunits, coordinates the membrane potential-dependent opening of the channel with the binding of the import motor on the matrix site via the membrane-associated subunit Tim44 (Meier et al., 2005; Martinez-Caballero et al., 2007; Ramesh et al., 2016; DemishteinZohary et al., 2017; Matta et al., 2017; Ting et al., 2017). Tim44 serves as binding site for the mitochondrial Hsp70 (mtHsp70) of the matrix which binds tightly to incoming polypeptides upon hydrolysis of its bound ATP to ADP in a reaction regulated by Pam18/Tim14 and Pam16/Tim16, a J protein and a J-like protein, respectively, that are associated with the TIM23 complex. Several binding reactions of $\mathrm{mtHsp} 70$ molecules are normally required to drive the complete translocation of a protein into the matrix. Mitochondrial presequences are removed in the matrix by the matrix processing peptidase. Preproteins may additionally be further processed by other enzymes before the mature proteins fold into their native structures. It should be noted that there appears to be no back-translocation across the inner membrane, so that matrix proteins stay in the matrix until they are degraded. This is different for IMS proteins, for which a back-translocation into the cytosol was reported under certain conditions (Bragoszewski et al., 2015).

Many presequence-containing inner membrane proteins are integrated into the inner membrane after translocational arrest by a transmembrane domain in their structure that serves as a stop-transfer sequence. Alternatively, inner membrane proteins can be initially completely imported into the matrix from where they insert into the inner membrane in an export-like reaction (Herrmann et al., 1997; Bohnert et al., 2010). Membrane insertion from the matrix is catalyzed by the Oxal protein of the inner membrane that is related to the bacterial insertase YidC (Hell et al., 2001). Some inner membrane proteins, such as members of the carrier family, lack presequences but insert into the inner membrane from the IMS using an alternative TIM translocase, the TIM22 complex (Sirrenberg et al., 1996; Hasson et al., 2010).

\section{ENERGETICS OF THE PROTEIN IMPORT INTO THE MITOCHONDRIAL MATRIX}

The mechanistic details of the translocation process across the TOM and the TIM23 were analyzed in many studies. However, our understanding of the molecular clockwork that drives protein translocation in vivo is still far from complete. This owes to the facts that (i) high-resolution structures of the import machinery are not available, (ii) the components of the import machinery interact in a highly dynamic fashion, and (iii) their interaction can only be analyzed in vitro or in organello as lysis even with mild detergents often leads to the loss of components and prevents the analysis of their functionality.

The initial translocation reactions from receptors on the mitochondrial surface via the TOM pore and IMS-exposed domains of the import machinery (Tom22, Tim50, Tim23) to the translocation pore of the TIM23 complex is presumably driven by the affinity of presequences to hydrophobic and negatively charged surfaces on components of the import machinery (Bolliger et al., 1995; Kanamori et al., 1997; Mokranjac et al., 2009; Shiota et al., 2015; Bausewein et al., 2017). Subsequently, the membrane potential across the inner membrane can further promote the translocation of the positively charged MTSs to the negatively charged, matrix side of the inner membrane (van der Laan et al., 2007). The inner membrane pore is gated by the presequences in a complicated process that involves an intricate interplay of Tim50, Tim23, and Tim17 (Meier et al., 2005; Martinez-Caballero et al., 2007; Schulz et al., 2011; Ramesh et al., 2016; Denkert et al., 2017; Schendzielorz et al., 2017). As soon as the presequence is exposed to the matrix, it can be bound by mitochondrial Hsp70 (mtHsp70) which completes matrix translocation of preproteins.

Two not mutually exclusive models were proposed, how mtHsp70 and other subunits of the mitochondrial import motor promote unidirectional translocation into the matrix (Figures 2A,B). The pros and cons of both models have been 

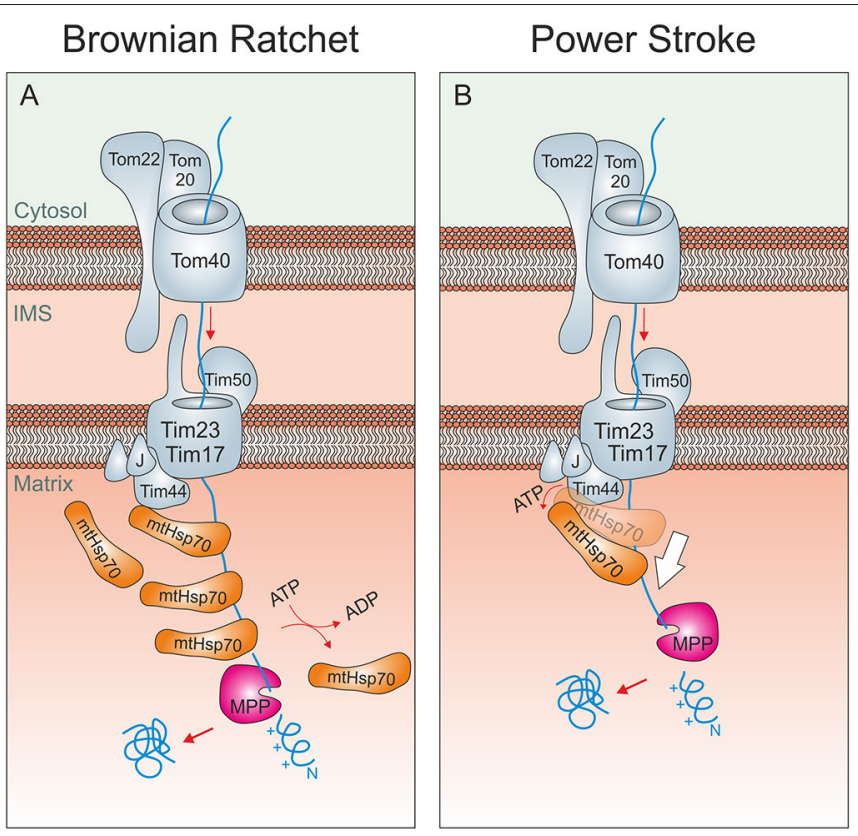

\section{Lateral Diffusion}

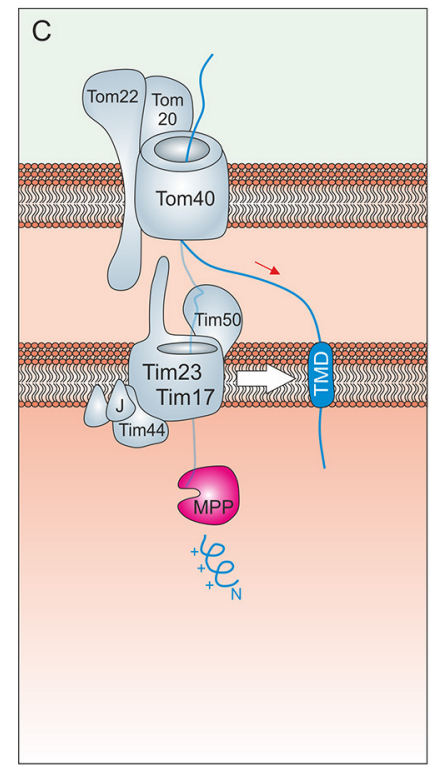

FIGURE 2 | Driving forces of mitochondrial protein import. (A) Brownian ratchet: According to this model, the ATP-hydrolysis does not promote a mechanical pulling of the incoming polypeptide. Rather, Hsp70 molecules associated to Tim44 bind to unfolded segments of the translocating protein, preventing their backsliding and thus rectifying their Brownian spontaneous motion into a vectorial movement into the matrix. (B) Power stroke: It was proposed that after binding to Tim44 and the presequence of an incoming preprotein, ATP-hydrolysis in Hsp70 triggers a large conformational change within the chaperone that leads to a mechanical pulling of the preprotein into the matrix. Repeated cycles would over time drive protein-translocation in a step-wise fashion. A pulling of Hsp70 was suggested to be particularly important if a folded domain on the mitochondrial surface needs/ed to be unfolded. It should be noted that both models are not mutually exclusive. (C) Lateral Diffusion: A number of inner membrane proteins contain stop-anchor sequences just C-terminal to their presequences. How their C-terminal domains are transported across the outer membrane is not known. It was suggested that lateral diffusion thus the separation of TOM and TIM23 complexes might be critical here.

extensively debated in many excellent studies and reviews in the past and will therefore not be repeated in detail here (Glick, 1995; Matlack et al., 1999; Voisine et al., 1999; Neupert and Brunner, 2002; Okamoto et al., 2002; Slutsky-Leiderman et al., 2007; Yamano et al., 2008). According to the Brownian ratchet model, repetitive binding of several $\mathrm{mtHsp} 70$ proteins prevents backsliding of intermediates. The energy for this translocation reaction initially comes from the Brownian movement of the incoming chain that is rectified by $\mathrm{mtHsp70}$. According to the alternative power stroke model, mtHsp70 actively pulls on the incoming polypeptides to drive their import in a step-wise fashion. Since the intramolecular movements of $\mathrm{mtHsp} 70$ are rather small, it is unlikely that they could processively run precursors into the matrix. However, small movements might help to unfold cytosolic domains of stalled import intermediates while the main driver of translocation is still rectified diffusion. From this perspective, the power stroke model can be seen as an extension rather than an alternative to the Brownian Ratchet model. The tight contact of mtHsp70 to the proteinconducting channel of the TIM23 translocase, which is critical for both mechanisms, is mediated by Tim44, Pam18/Tim14, and Pam16/Tim16 (Demishtein-Zohary et al., 2017; Matta et al., 2017; Ting et al., 2017).

An interesting variation of the Brownian Ratchet model was recently proposed (De Los Rios et al., 2006; Finka et al., 2015). Though this model is called entropic pulling, no power stroke is involved here. Rather, changes in the conformational freedom of the components of the import motor and the incoming polypeptide forcefully drive the unfolding and translocation of precursor proteins in an ATP-dependent reaction. Whether the hypothesis of entropic pulling really settles the debate between translocation by Brownian Ratchet and by power stroke is still open. However, it certainly is very attractive since it naturally integrates the ratcheting property of the Brownian Ratchet model and the active pulling action of the power stroke model in a single framework. The import motor is unable to drive the translocation of inner membrane proteins with amino terminal transmembrane domains. It is not clear how C-terminal domains of these proteins are driven across the TOM complex but it was proposed that the lateral diffusion of the transmembrane domains drives this process (Figure 2C). Nonetheless, it is difficult to envision how diffusion alone shall be sufficient to unfold domains in the cytosol in order to allow their passage through the TOM pore. This is one of the open questions in the field that still awaits to be answered.

\section{THE MITOCHONDRIAL DISULFIDE RELAY}

The IMS is a small compartment that is enclosed by the outer and inner membrane of mitochondria. Proteomic studies identified about 50 and 130 different IMS proteins in yeast and mammalian 
mitochondria, respectively (Vögtle et al., 2012; Hung et al., 2014). Some of these proteins, particularly those of larger mass and multi-domain organization are synthesized with "bipartite presequences," i.e., amino terminal targeting signals consisting of an MTS followed by a stop-transfer sequence that is cleaved off after translocation thus giving rise to a mature soluble IMS protein. Examples for IMS proteins with bipartite presequences include proapoptotic factors such as Smac/Diablo (Burri et al., 2005), apoptosis-inducing factor (AIF) (Hangen et al., 2015), or endonuclease $\mathrm{G}$ (Ohsato et al., 2002) as well as enzymes such as cytochrome $b_{2}$ (Glick et al., 1992), Mgm1 (Herlan et al., 2004), or cytochrome $c$ peroxidase (Michaelis et al., 2005).

Most proteins of the IMS lack presequences and their amino termini do not share any characteristics with those of matrix proteins. Many of these proteins are of relatively low mass $(7-25 \mathrm{kDa})$ and of rather simple structure (most frequent is a simple helix-loop-helix organization). Many of these proteins appear to make use of high-affinity binding sites in the IMS that are crucial for their translocation through the proteinconducting channel of the TOM complex. The most abundant IMS protein, cytochrome $c$, employs its hemylating enzyme cytochrome $c$ heme lyase as a trans-site receptor (Nargang et al., 1988; Nicholson and Neupert, 1989; Dumont et al., 1991). Apocytochrome $c$ can cross the outer membrane through the TOM complex in both directions. In the IMS, cytochrome $c$ heme lyase incorporates a heme group into apocytochrome $c$ and thereby triggers its stable folding rendering holocytochrome $c$ unable to retro-translocate through the TOM complex. In this reaction, cytochrome $c$ heme lyase functions both as a receptor and as a converting enzyme that catalyzes the stable folding of cytochrome $c$.

Mechanistically, the function of cytochrome $c$ heme lyase for the import of cytochrome $c$ is presumably similar to that of Mia40, a highly conserved IMS proteins found in mitochondria of plants, fungi, animals and humans. Mia40 is an oxidoreductase that can introduce disulfide bonds into its substrates and most IMS proteins indeed contain disulfide bonds (Gabriel et al., 2007; Longen et al., 2009; Kawamata and Manfredi, 2010; Klöppel et al., 2011; Vögtle et al., 2012; Kritsiligkou et al., 2017). Mia40 was initially identified in mitochondria of budding yeast (Sickmann et al., 2003; Chacinska et al., 2004; Naoe et al., 2004) before other orthologs were discovered. Mia40 is also referred to as Tim40 (in yeast) and as CHCHD4 (in mammalian cells). All Mia40 homologs share a highly conserved central region containing six invariant cysteine residues. A redox-sensitive CPC motif is essential for the oxidoreductase activity of Mia40. At steady state, this CPC is predominantly present in the oxidized state, although the degree of Mia40 oxidation might vary between different organisms and prevailing redox conditions (Bien et al., 2010; Sztolsztener et al., 2013; Kojer et al., 2014). The cysteine residues form intermolecular disulfide bonds with Mia40 substrates that can be stable for several minutes (Chacinska et al., 2004; Naoe et al., 2004; Mesecke et al., 2005; Longen et al., 2009; Sideris et al., 2009; Koch and Schmid, 2014c) and, at least in vitro, can promote both oxidation and isomerization reactions (Weckbecker et al., 2012; Koch and Schmid, 2014a). Erv1 (ALR in humans) is a FAD-bound sulfhydryl oxidase in the IMS that maintains Mia40 in its active, oxidized state (Lisowsky, 1994; Allen et al., 2005; Mesecke et al., 2005; Rissler et al., 2005; Terziyska et al., 2005; Ang and Lu, 2009; Tienson et al., 2009). Erv1 can either directly reduce oxygen to hydrogen peroxide or use cytochrome $c$ as an electron acceptor (Figure 3). Alternatively, it can interact with the fumarate reductase Osm 1 in order to get re-oxidized under anaerobic conditions (Neal et al., 2017).

\section{MECHANISMS OF MIA40-MEDIATED PROTEIN IMPORT INTO THE IMS}

The CPC motif of Mia40 is in direct proximity to its hydrophobic substrate-binding pocket, which is formed by two anti-parallel helices stabilized by two structural disulfide bonds (Banci et al., 2009; Kawano et al., 2009). Mia40 substrates reach the IMS via translocation through the TOM pore and already bind to Mia40 during their translocation (von der Malsburg et al., 2011; Banci et al., 2012; Peleh et al., 2016). Mia40 recognizes specific patterns in its substrates referred to as intermembrane space targeting signal (ITS) or mitochondrial IMS-sorting signal (MISS) (Milenkovic et al., 2009; Sideris et al., 2009). However, the specificity of the Mia40 binding might be rather low since, at least in vitro, Mia40 interacts rather generally with hydrophobic protein stretches, particularly if they are of helical nature (Koch and Schmid, 2014c). In this reaction, Mia40 serves as a receptor that facilitates protein translocation by substrate trapping (Peleh et al., 2016). The interaction of Mia40 with its substrate can last from several seconds to minutes, and it was suggested that this prevents the back-translocation of Mia40 substrates into the cytosol.

Mia40 substrates are released into the IMS in an oxidized state. The formation of disulfide bonds locks these proteins in a stably

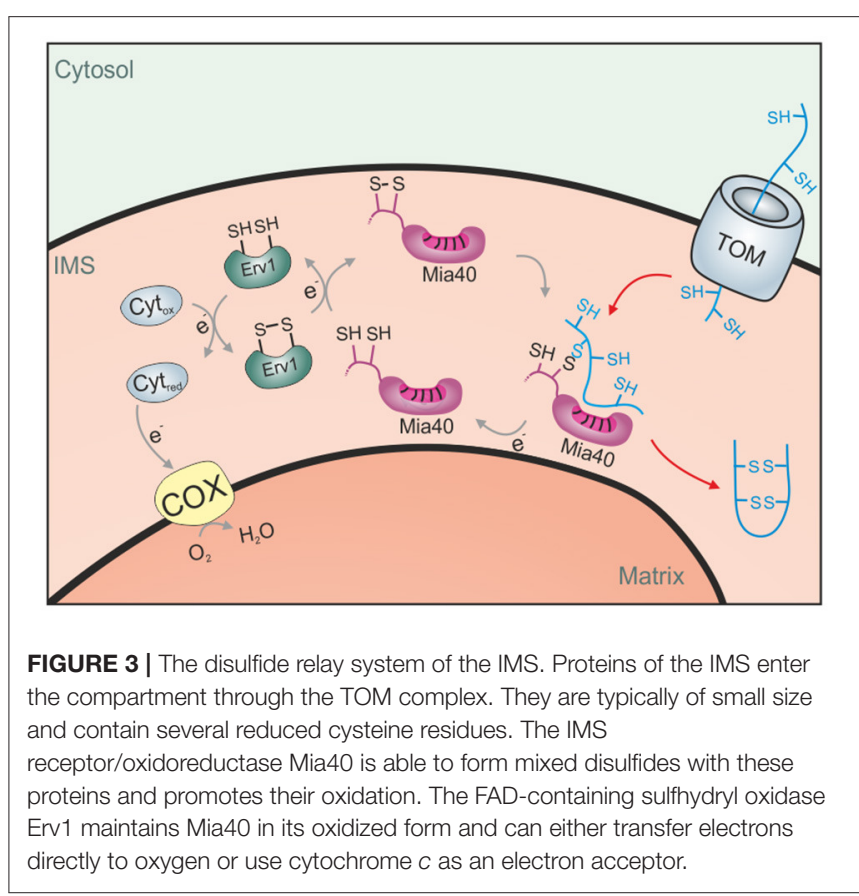


folded conformation thereby trapping them in the IMS as the folded proteins cannot pass the protein-conducting channel of the TOM complex (Sideris and Tokatlidis, 2007; Morgan and Lu, 2008; Bragoszewski et al., 2015). Initially, a "folding trap hypothesis" was proposed suggesting that newly synthesized IMS proteins would diffuse into and out of the IMS unless their oxidation by Mia40 keeps them within mitochondria (Allen et al., 2003; Lutz et al., 2003; Figure 4A). Indeed, even fully imported, endogenous IMS proteins were found to be released from the IMS through the TOM pore if their structural disulfide bonds are reduced by the addition of reductants (Bragoszewski et al., 2015). More detailed analyses of the import process and the use of site-specific cysteine mutants indicated that Mia40 serves as a molecular trap that binds incoming polypeptides via disulfide bonds (Figure 4B) to mediate their translocation through the TOM pore (Milenkovic et al., 2009; Sideris et al., 2009; von der Malsburg et al., 2011; Banci et al., 2012; Koch and Schmid, 2014c). However, this model was challenged by the observation that the redox-active CPC motif of Mia40 is dispensable for IMS import and only crucial subsequently for substrate folding (Baker et al., 2012; Weckbecker et al., 2012; Wrobel et al., 2013; Peleh et al., 2016; Ramesh et al., 2016). Thus, Mia40 can initially serve as trans-site receptor or holdase that promotes protein translocation across the TOM pore in an oxidation-independent process (Figure 4C).

However, Mia40 is certainly more than a simple receptor protein as it serves as a chaperone with foldase activity for a number of substrate proteins, a function which can even be observed for substrate proteins that do not contain cysteine residues (Weckbecker et al., 2012). The oxidative folding in the IMS is facilitated by glutathione (Bien et al., 2010; Kojer et al., 2012, 2014) and a number of redox enzymes, such as the peroxidase Gpx3 or thioredoxins, however, their specific contribution to the oxidative folding process is still not well understood (Vögtle et al., 2012; Kritsiligkou et al., 2017). In mammalian mitochondria, Mia40 forms a complex with the oxidoreductase apoptosis inducing factor (AIF) which tethers Mia40 to the inner membrane (Hangen et al., 2015).

The three different mechanisms shown in Figure 4 are not mutually exclusive. Mia40 obviously is able to trap incoming polypeptides both by its hydrophobic interaction to their MISS/ITS signal and by the formation of mixed disulfides. The observation that oxidative folding is critical to maintain some IMS proteins stable in the IMS certainly also argues for an oxidative folding trap function that is relevant for IMS proteins. The contribution of each of these mechanisms to the import process might also differ between substrates and physiological conditions.

\section{ENERGETICS OF THE MIA40-MEDIATED PROTEIN IMPORT INTO THE IMS}

Little is known about the mechanistic steps that drive the translocation of substrates of the mitochondrial disulfide relay across the outer membrane. There is no evidence for a cotranslational import of IMS proteins in which the translation on the ribosome could promote the translocation through the TOM complex. As long as translation is not inhibited by antibiotics almost no cytosolic ribosomes are associated with the mitochondrial outer membrane (Gold et al., 2017).

Recent studies showed that the binding to Mia40 is essential for the translocation reaction but the oxidation of cysteines is not. This is supported by the observation that mutants of the Mia40 substrates Atp23, Tim9, and Tim10 accumulate in the IMS even if all cysteine residues are mutated (Baker et al., 2012; Weckbecker et al., 2012). However, these mutated proteins are rapidly degraded by the i-AAA protease Yme1 in the IMS. Moreover, a Mia40 mutant lacking the CPC motif still mediates protein import into the IMS, thus the oxidoreductase activity is not essential for its role as import component (Peleh et al., 2016). Mia40 might drive the import reaction by a lateral diffusionmediated process (Figure 5A) similar to the process that drives import of inner membrane proteins (Figure 2C). Alternatively, Mia40 could simply act as a rectifier of diffusion by preventing backsliding of the substrate, comparable to the role of $\mathrm{mtHsp70}$ in the Brownian Ratchet of the matrix (Figure 2A).

It is also feasible that the folding of substrate proteins by Mia40, for example due to the oxidation of substrates, promotes the compaction of IMS proteins and helps to translocate the termini of IMS proteins through the TOM pore (Figure 5B). However, such a process would presumably only be able to drive the translocation of relatively short segments into the IMS.

\section{THE MECHANISM OF MIA40-MEDIATED PROTEIN IMPORT CONSTRAINS THE PROPERTIES OF IMS PROTEINS}

Even if the details are still not clear, it is obvious that in respect to its energetics, the Mia40-driven import differs considerably from the $\mathrm{mtHsp} 70$-driven translocation of matrix proteins which might explain several obvious differences in the molecular nature of matrix and IMS proteins:

\section{Mia40 Substrates Show a Strong Bias Toward Small Size}

The masses of most Mia40 substrates are extremely low (for example: Cmc1, $13.0 \mathrm{kDa}$; Cox17, $8.0 \mathrm{kDa}$; Cox19, $11.1 \mathrm{kDa}$ Mdm35, 9.7 kDa; Mic14, 13.8 kDa; Mrp10, 9.7 kDa; Tim8, 9.7 kDa; Tim9, $10.2 \mathrm{kDa}$; Tim10, $10.3 \mathrm{kDa}$; Tim12, $12.3 \mathrm{kDa}$; Tim13, $11.3 \mathrm{kDa}$ ). Many of these proteins have $<100$ amino acid residues and therefore were initially not even annotated when the yeast genome was sequenced. The largest known soluble Mia40 substrate, Atp23 (32.2 kDa), differs from all other substrates since it has five rather than only two structural disulfide bonds and might employ several Mia40 molecules to be imported (Weckbecker et al., 2012; Kojer et al., 2014). In contrast, matrix proteins can be large and often of several distinct folding units (e.g., Pim1, 127 kDa; Kgd1, 114 kDa; Pet309, 113 kDa). The processive $\mathrm{mtHsp} 70$-mediated translocation obviously can easily mediate the import of polypeptides that consist of many hundreds of amino acid residues, but Mia40 might not. The small size of IMS proteins is not explained by the tiny lumen of this 


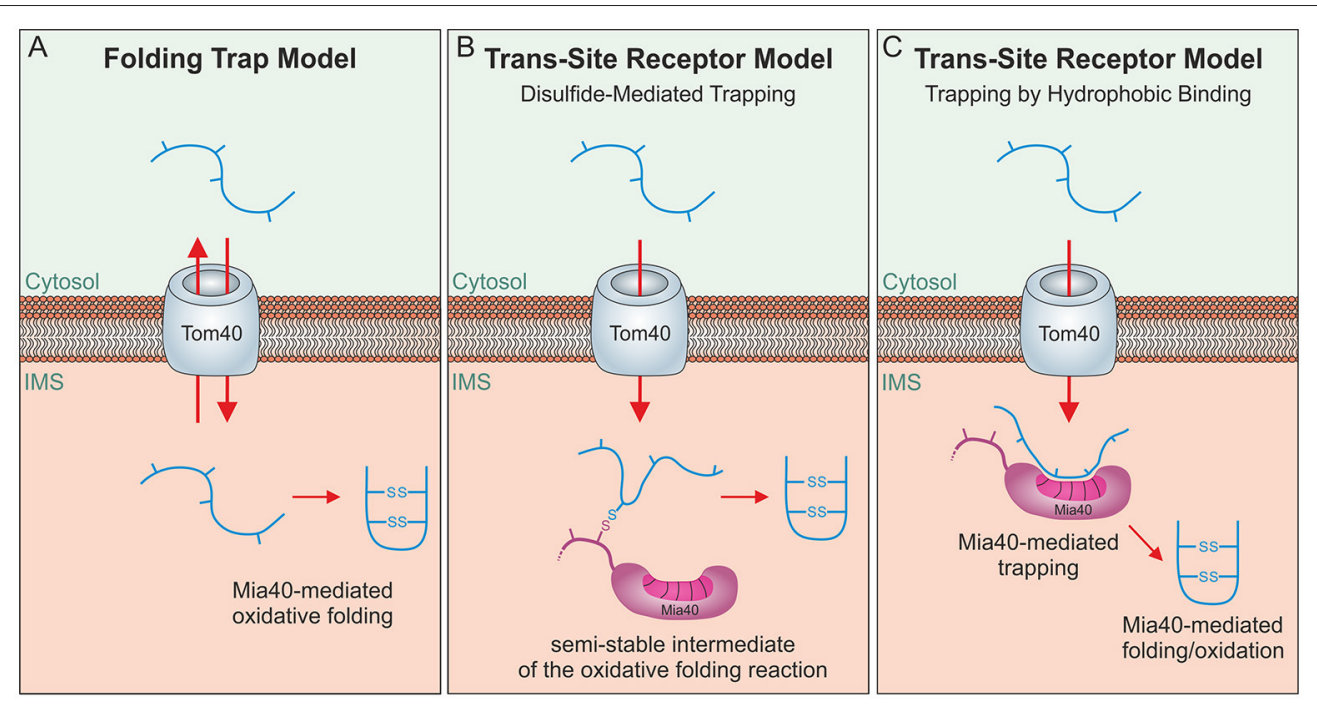

FIGURE 4 | Different models of oxidative folding by Mia40. (A) Folding Trap Model: This model was inspired by the observation that reduced IMS proteins can back-translocate from the IMS to the cytosol. Since Mia40-mediated folding prevents this back-translocation, it was proposed that Mia40 does not directly promote translocation across the membrane but rather traps IMS proteins that were imported by facilitated diffusion through the TOM complex. (B) Disulfide-mediated trapping: Mia40 binds incoming proteins through mixed disulfides to prevent their backsliding into the cytosol and thus serves as a trans-site receptor that functions in a redox-mediated manner. (C) Trapping by hydrophobic binding: Mia40 is able to serve as a trans-site receptor that can mediate protein translocation in an oxidation-independent manner using hydrophobic interactions with the MIS/ITS signals in their sequence.
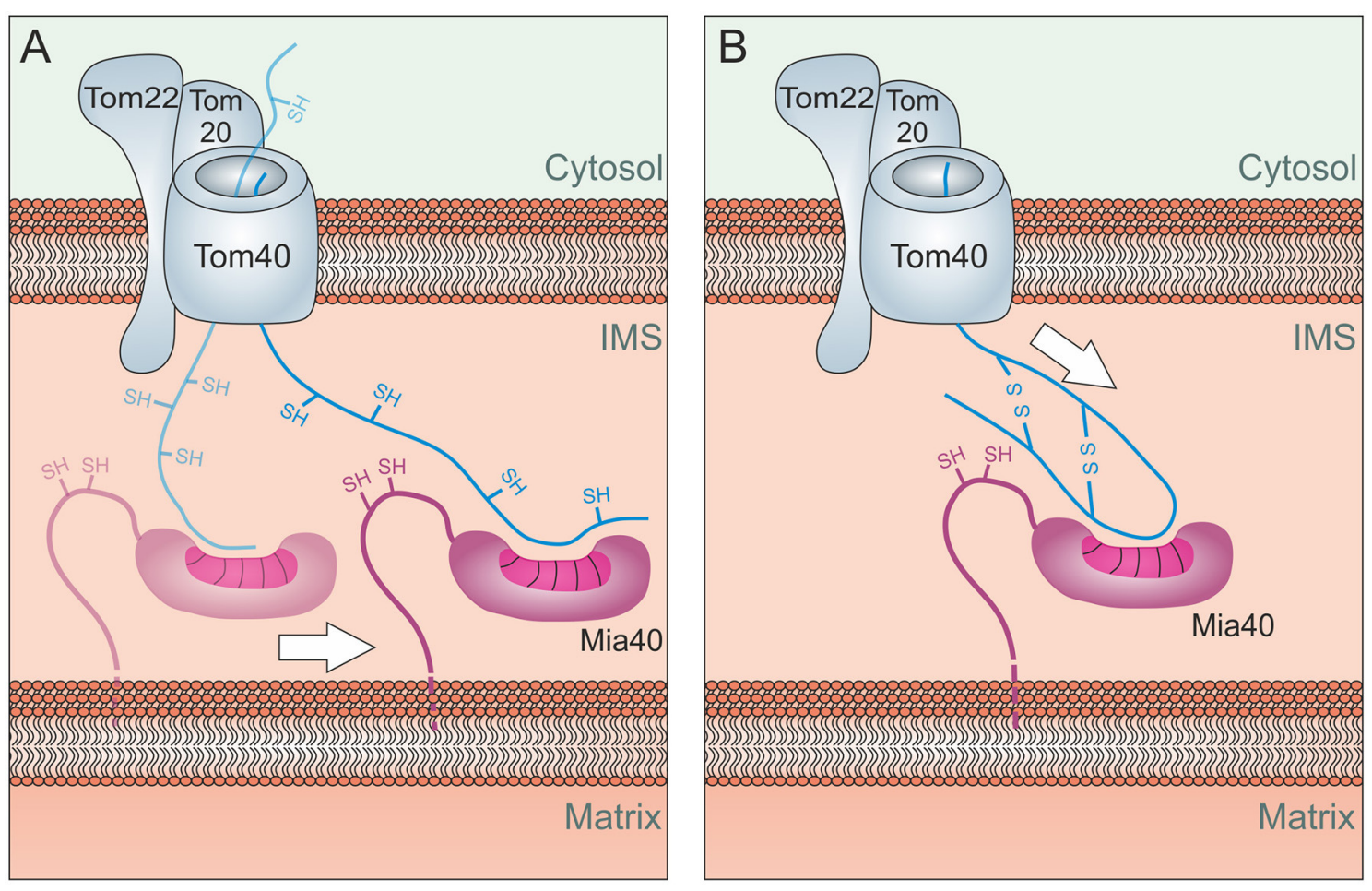

FIGURE 5 | Energetics of Mia40-mediated import. (A) Lateral Diffusion: Mia40 binds to the translocating protein via hydrophobic binding which might be further stabilized by mixed disulfide bonds with the protein. Through lateral diffusion, Mia40 might drive the import reaction into the IMS. (B) Oxidation-mediated compaction: The oxidation of the incoming proteins and hence their compaction might contribute to the driving of the import process. Such a process would be limited to the import of short sequences across the TOM pore, explaining why most Mia40 substrates are of very small size. 
compartment since a number of IMS-exposed proteins that use presequences are much larger than Mia40 substrates (Yme1, 81.1 $\mathrm{kDa}$; cytochrome $\mathrm{b}_{2}, 65.5 \mathrm{kDa}$ ).

\section{Mia40 Substrates Are of Simple Helix-Loop-Helix Structure}

Almost all Mia40 substrates identified so far are consist of two short $\alpha$-helices that are connected by two parallel disulfide bonds. Whereas substrates of the thioredoxin-based oxidation machineries of the ER and the periplasm are of very diverse structure, Mia40 substrates have a surprisingly consistent fold. This might be due to the poor catalytic capacity of Mia40 in oxidation and isomerisation reactions (Koch and Schmid, 2014a,b,c) but also might be used to drive the full translocation of proteins into the IMS during the oxidation process (Figure 5B). Obviously, such a process could only promote the translocation of a short stretch, thus explaining the small size of most IMS proteins.

\section{Mia40 Substrates Show Only a Low Affinity for Mitochondrial Surface Receptors}

The receptors of the TOM complex efficiently bind to mitochondrial presequences but show only very low affinity to Mia40 substrates (Lutz et al., 2003). Instead of the cytosolexposed TOM receptors, Tom70, Tom22, and Tom20, IMS proteins directly bind to the pore-forming subunit Tom40 and to the small TOM protein Tom5 (Kurz et al., 1999; Vögtle et al., 2012; Gornicka et al., 2014). It is unclear why IMS proteins avoid high-affinity interactions to the TOM complex, but it seems conceivable that the Mia40-dependent import process does not provide the energy required to release preproteins from the TOM receptors since this pathway functions independent of potent energy sources such as ATP hydrolysis or the membrane potential.

Apparently, the mitochondrial IMS is a very unique compartment in the cell as the limitations of the Mia40mediated import systems constrained the properties of its proteome. The biochemical functions of many of the small helix-loop-helix proteins of the IMS are not understood and it will be very interesting to understand whether their common overall structure restricts their function to one common overall biochemical activity. However, one common type of reaction carried out by all of these different proteins is difficult to reconcile, given the many roles that these proteins exhibit in lipid homeostasis, respiratory chain complex assembly, or the transfer of copper ions.

\section{REFERENCES}

Aaltonen, M. J., Friedman, J. R., Osman, C., Salin, B., di Rago, J. P., Nunnari, J., et al. (2016). MICOS and phospholipid transfer by Ups2-Mdm35 organize membrane lipid synthesis in mitochondria. J. Cell Biol. 213, 525-534. doi: $10.1083 /$ jcb. 201602007

Allen, S., Balabanidou, V., Sideris, D. P., Lisowsky, T., and Tokatlidis, K. (2005). Erv1 mediates the Mia40-dependent protein import pathway and provides a

\section{FINAL REMARKS}

As far as we know the matrix-targeting machinery is very similar in different eukaryotes. There are certainly differences in the TOM receptors, which are not well conserved, however, the major components of the TOM and TIM23 complexes, as well as the import motor are conserved. In contrast, the mitochondrial disulfide relay differs considerably among eukaryotes and many protists lack a Mia40 homolog (though they contain an Erv1 protein). Also in plants, Erv1 can directly bind substrates making Mia40 dispensable. Unfortunately, little is known about the mechanisms of protein translocation in these organisms and other, so far not characterized factors might take over the holdase and foldase function of Mia40 (Carrie et al., 2010; Eckers et al., 2013; Haindrich et al., 2017; Peleh et al., 2017).

The mitochondrial intermembrane space developed from the bacterial periplasm during evolution. This common origin might explain the presence of disulfide bonds in most IMS proteins. The periplasm does not contain ATP (to avoid its loss by diffusion through porins of the outer membrane) which might have forced bacteria to develop a mechanism to fold periplasmic proteins by chaperones that act independently of ATP hydrolysis. DsbA, the thioredoxin that introduces disulfide bonds into periplasmic proteins, is one of these folding factors. The IMS is one of the very few eukaryotic compartments which, as far as we know, does not contain an Hsp70 chaperone system, perhaps because the early eukaryotic cells managed to exploit its oxidation machinery to drive protein translocation. Since the mitochondrial disulfide relay is of much lower complexity than the TIM23 import motor it might be possible to design a reconstituted system that can drive protein translocation. This certainly would be a big step forward in order to better understand its mechanistic properties in mitochondrial protein biogenesis.

\section{AUTHOR CONTRIBUTIONS}

All authors listed have made a substantial, direct and intellectual contribution to the work, and approved it for publication.

\section{FUNDING}

The research on the mitochondrial disulfide relay in the authors' laboratory is financed by grants from the Deutsche Forschungsgemeinschaft (SPP1710; IRTG1830; He2803/4-2). J. Mol. Biol. 353, 937-944. doi: 10.1016/j.jmb.2005.08.049

Allen, S., Lu, H., Thornton, D., and Tokatlidis, K. (2003). Juxtaposition of the two distal CX3C motifs via intrachain disulfide bonding is essential for the folding of Tim10. J. Biol. Chem. 278, 38505-38513. doi: 10.1074/jbc.M306027200

Ang, S. K., and Lu, H. (2009). Deciphering structural and functional roles of individual disulfide bonds of the mitochondrial sulfhydryl oxidase Ervlp. J. Biol. Chem. 284, 28754-28761. doi: 10.1074/jbc.M109.021113 
Bajaj, R., Jaremko, L., Jaremko, M., Becker, S., and Zweckstetter, M. (2014). Molecular basis of the dynamic structure of the TIM23 complex in the mitochondrial intermembrane space. Structure 22, 1501-1511. doi: 10.1016/j.str.2014.07.015

Baker, M. J., Mooga, V. P., Guiard, B., Langer, T., Ryan, M. T., and Stojanovski, D. (2012). Impaired folding of the mitochondrial small TIM chaperones induces clearance by the i-AAA protease. J. Mol. Biol. 424, 227-239. doi: 10.1016/j.jmb.2012.09.019

Banci, L., Bertini, I., Cefaro, C., Ciofi-Baffoni, S., Gallo, A., Martinelli, M., et al. (2009). Mia40 is an oxidoreductase that catalyzes oxidative protein folding in mitochondria. Nat. Struct. Mol. Biol. 16, 198-206. doi: 10.1038/nsmb.1553

Banci, L., Bertini, I., Ciofi-Baffoni, S., Jaiswal, D., Neri, S., Peruzzini, R., et al. (2012). Structural characterization of CHCHD5 and CHCHD7: two atypical human twin CX9C proteins. J. Struct. Biol. 180, 190-200. doi: 10.1016/j.jsb.2012.07.007

Bausewein, T., Mills, D. J., Langer, J. D., Nitschke, B., Nussberger, S., and Kuhlbrandt, W. (2017). Cryo-EM structure of the TOM core complex from Neurospora crassa. Cell 170, 693.e7-700 e7. doi: 10.1016/j.cell.2017.07.012

Bien, M., Longen, S., Wagener, N., Chwalla, I., Herrmann, J. M., and Riemer, J. (2010). Mitochondrial disulfide bond formation is driven by intersubunit electron transfer in Erv1 and proof read by glutathione. Mol. Cell 37, 516-528. doi: 10.1016/j.molcel.2010.01.017

Bohnert, M., Rehling, P., Guiard, B., Herrmann, J. M., Pfanner, N., and van der Laan, M. (2010). Cooperation of stop-transfer and conservative sorting mechanisms in mitochondrial protein transport. Curr. Biol. 20, 1227-1232. doi: 10.1016/j.cub.2010.05.058

Bolliger, L., Junne, T., Schatz, G., and Lithgow, T. (1995). Acidic receptor domains on both sides of the outer membrane mediate translocation of precursor proteins into yeast mitochondria. EMBO J. 14, 6318-6326.

Bragoszewski, P., Wasilewski, M., Sakowska, P., Gornicka, A., Bottinger, L., Qiu, J., et al. (2015). Retro-translocation of mitochondrial intermembrane space proteins. Proc. Natl. Acad. Sci. U.S.A. 112, 7713-7718. doi: 10.1073/pnas.1504615112

Brix, J., Rudiger, S., Bukau, B., Schneider-Mergener, J., and Pfanner, N. (1999). Distribution of binding sequences for the mitochondrial import receptors Tom20, Tom22, and Tom70 in a presequence-carrying preprotein and a non-cleavable preprotein. J. Biol. Chem. 274, 16522-16530. doi: $10.1074 /$ jbc.274.23.16522

Burri, L., Strahm, Y., Hawkins, C. J., Gentle, I. E., Puryer, M. A., Verhagen, A., et al. (2005). Mature DIABLO/Smac is produced by the IMP protease complex on the mitochondrial inner membrane. Mol. Biol. Cell 16, 2926-2933. doi: 10.1091/mbc.E04-12-1086

Calvo, S. E., Clauser, K. R., and Mootha, V. K. (2016). MitoCarta2.0: an updated inventory of mammalian mitochondrial proteins. Nucleic Acids Res. 44, D1251D1257. doi: $10.1093 / \mathrm{nar} / \mathrm{gkv} 1003$

Calvo, S. E., Julien, O., Clauser, K. R., Shen, H., Kamer, K. J., Wells, J. A., et al. (2017). Comparative analysis of mitochondrial N-termini from mouse, human, and yeast. Mol. Cell. Proteomics 16, 512-523. doi: 10.1074/mcp.M116. 063818

Carrie, C., Giraud, E., Duncan, O., Xu, L., Wang, Y., Huang, S., et al. (2010). Conserved and novel functions for Arabidopsis thaliana MIA40 in assembly of proteins in mitochondria and peroxisomes. J. Biol. Chem. 285, 36138-36148. doi: 10.1074/jbc.M110.121202

Chacinska, A., Pfannschmidt, S., Wiedemann, N., Kozjak, V., Sanjuan Szklarz, L. K., Schulze-Specking, A., et al. (2004). Essential role of Mia40 in import and assembly of mitochondrial intermembrane space proteins. EMBO J. 23, 3735-3746. doi: 10.1038/sj.emboj.7600389

De Los Rios, P., Ben-Zvi, A., Slutsky, O., Azem, A., and Goloubinoff, P. (2006). Hsp70 chaperones accelerate protein translocation and the unfolding of stable protein aggregates by entropic pulling. Proc. Natl. Acad. Sci. U.S.A. 103, 6166-6171. doi: 10.1073/pnas.0510496103

Demishtein-Zohary, K., Gunsel, U., Marom, M., Banerjee, R., Neupert, W., Azem, A., et al. (2017). Role of Tim17 in coupling the import motor to the translocation channel of the mitochondrial presequence translocase. Elife 6:e22696. doi: 10.7554/eLife.22696

Denkert, N., Schendzielorz, A. B., Barbot, M., Versemann, L., Richter, F., Rehling, P., et al. (2017). Cation selectivity of the presequence translocase channel Tim 23 is crucial for efficient protein import. Elife 6:e28324. doi: 10.7554/eLife.28324
Dumont, M. E., Cardillo, T. S., Hayes, M. K., and Sherman, F. (1991). Role of cytochrome $\mathrm{c}$ heme lyase in mitochondrial import and accumulation of cytochrome c in Saccharomyces cerevisiae. Mol. Cell. Biol. 11, 5487-5496. doi: 10.1128/MCB.11.11.5487

Eckers, E., Petrungaro, C., Gross, D., Riemer, J., Hell, K., and Deponte, M. (2013). Divergent molecular evolution of the mitochondrial sulfhydryl:cytochrome C oxidoreductase Erv in opisthokonts and parasitic protists. J. Biol. Chem. 288, 2676-2688. doi: 10.1074/jbc.M112.420745

Fan, A. C., Kozlov, G., Hoegl, A., Marcellus, R. C., Wong, M. J., Gehring, K., et al. (2011). Interaction between the human mitochondrial import receptors Tom 20 and Tom70 in vitro suggests a chaperone displacement mechanism. J. Biol. Chem. 286, 32208-32219. doi: 10.1074/jbc.M111.280446

Finka, A., Sharma, S. K., and Goloubinoff, P. (2015). Multi-layered molecular mechanisms of polypeptide holding, unfolding and disaggregation by HSP70/HSP110 chaperones. Front. Mol. Biosci. 2:29. doi: $10.3389 /$ fmolb.2015.00029

Gabriel, K., Milenkovic, D., Chacinska, A., Muller, J., Guiard, B., Pfanner, N., et al. (2007). Novel mitochondrial intermembrane space proteins as substrates of the MIA import pathway. J. Mol. Biol. 365, 612-620. doi: 10.1016/j.jmb.2006.10.038

Glick, B. S. (1995). Can Hsp70 proteins act as force-generating motors? Cell 80, 11-14.

Glick, B. S., Brandt, A., Cunningham, K., Muller, S., Hallberg, R. L., and Schatz, G. (1992). Cytochromes $\mathrm{c} 1$ and b2 are sorted to the intermembrane space of yeast mitochondria by a stop-transfer mechanism. Cell 69, 809-822. doi: 10.1016/0092-8674(92)90292-K

Gold, V. A., Chroscicki, P., Bragoszewski, P., and Chacinska, A. (2017). Visualization of cytosolic ribosomes on the surface of mitochondria by electron cryo-tomography. EMBO Rep. 18, 1786-1800. doi: 10.15252/embr.201744261

Gornicka, A., Bragoszewski, P., Chroscicki, P., Wenz, L. S., Schulz, C., Rehling, P., et al. (2014). A discrete pathway for the transfer of intermembrane space proteins across the outer membrane of mitochondria. Mol. Biol. Cell 25, 3999-4009. doi: 10.1091/mbc.E14-06-1155

Haindrich, A. C., Boudova, M., Vancova, M., Diaz, P. P., Horakova, E., and Lukes, J. (2017). The intermembrane space protein Erv1 of Trypanosoma brucei is essential for mitochondrial Fe-S cluster assembly and operates alone. Mol. Biochem. Parasitol. 214, 47-51. doi: 10.1016/j.molbiopara.2017.03.009

Hangen, E., Feraud, O., Lachkar, S., Mou, H., Doti, N., Fimia, G. M., et al. (2015). Interaction between AIF and $\mathrm{CHCHD} 4$ regulates respiratory chain biogenesis. Mol. Cell 58, 1001-1014. doi: 10.1016/j.molcel.2015.04.020

Hansen, K. G., Schlagowski, A., and Herrmann, J. M. (2016). Escorted by chaperones: Stil helps to usher precursor proteins from the ribosome to mitochondria. FEBS J. 283, 3335-3337. doi: 10.1111/febs.13821

Hasson, S. A., Damoiseaux, R., Glavin, J. D., Dabir, D. V., Walker, S. S., and Koehler, C. M. (2010). Substrate specificity of the TIM22 mitochondrial import pathway revealed with small molecule inhibitor of protein translocation. Proc. Natl. Acad. Sci. U.S.A. 107, 9578-9583. doi: 10.1073/pnas.0914387107

Hell, K., Neupert, W., and Stuart, R. A. (2001). Oxalp acts as a general membrane insertion machinery for proteins encoded by mitochondrial DNA. EMBO J. 20, 1281-1288. doi: 10.1093/emboj/20.6.1281

Herlan, M., Bornhovd, C., Hell, K., Neupert, W., and Reichert, A. S. (2004). Alternative topogenesis of Mgm1 and mitochondrial morphology depend on ATP and a functional import motor. J. Cell Biol. 165, 167-173. doi: $10.1083 /$ jcb.200403022

Herrmann, J. M., Neupert, W., and Stuart, R. A. (1997). Insertion into the mitochondrial inner membrane of a polytopic protein, the nuclear encoded Oxalp. EMBO J. 16, 2217-2226. doi: 10.1093/emboj/16.9.2217

Herrmann, J. M., and Riemer, J. (2010). The intermembrane space of mitochondria. Antioxid. Redox Signal. 13, 1341-1358. doi: $10.1089 /$ ars.2009.3063

Hoseini, H., Pandey, S., Jores, T., Schmitt, A., Franz-Wachtel, M., Macek, B., et al. (2016). The cytosolic cochaperone Stil is relevant for mitochondrial biogenesis and morphology. FEBS J. 283, 3338-3352. doi: 10.1111/febs.13813

Hung, V., Zou, P., Rhee, H. W., Udeshi, N. D., Cracan, V., Svinkina, T., et al. (2014). Proteomic mapping of the human mitochondrial intermembrane space in live cells via ratiometric APEX tagging. Mol. Cell 55, 332-341. doi: 10.1016/j.molcel.2014.06.003

Kanamori, T., Nishikawa, S., Shin, I., Schultz, P. G., and Endo, T. (1997). Probing the environment along the protein import pathways in yeast mitochondria 
by site-specific photocrosslinking. Proc. Natl. Acad. Sci. U.S.A. 94, 485-490. doi: 10.1073/pnas.94.2.485

Kawamata, H., and Manfredi, G. (2010). Import, maturation, and function of SOD1 and its copper chaperone CCS in the mitochondrial intermembrane space. Antioxid. Redox Signal. 13, 1375-1384. doi: 10.1089/ars.2010.3212

Kawano, S., Yamano, K., Naoe, M., Momose, T., Terao, K., Nishikawa, S., et al. (2009). Structural basis of yeast Tim40/Mia40 as an oxidative translocator in the mitochondrial intermembrane space. Proc. Natl. Acad. Sci. U.S.A. 106, 14403-14407. doi: 10.1073/pnas.0901793106

Klöppel, C., Suzuki, Y., Kojer, K., Petrungaro, C., Longen, S., Fiedler, S., et al. (2011). Mia40-dependent oxidation of cysteines in domain I of Ccs1 controls its distribution between mitochondria and the cytosol. Mol. Biol. Cell 22, 3749-3757. doi: 10.1091/mbc.E11-04-0293

Koch, J. R., and Schmid, F. X. (2014a). Mia40 combines thiol oxidase and disulfide isomerase activity to efficiently catalyze oxidative folding in mitochondria. $J$. Mol. Biol. 426, 4087-4098. doi: 10.1016/j.jmb.2014.10.022

Koch, J. R., and Schmid, F. X. (2014b). Mia40 is optimized for function in mitochondrial oxidative protein folding and import. ACS Chem. Biol. 9, 2049-2057. doi: 10.1021/cb500408n

Koch, J. R., and Schmid, F. X. (2014c). Mia40 targets cysteines in a hydrophobic environment to direct oxidative protein folding in the mitochondria. Nat. Commun. 5:3041. doi: 10.1038/ncomms4041

Kojer, K., Bien, M., Gangel, H., Morgan, B., Dick, T. P., and Riemer, J. (2012). Glutathione redox potential in the mitochondrial intermembrane space is linked to the cytosol and impacts the Mia40 redox state. EMBO J. 31, 3169-3182. doi: 10.1038/emboj.2012.165

Kojer, K., Peleh, V., Calabrese, G., Herrmann, J. M., and Riemer, J. (2014). Kinetic control by limiting glutaredoxin amounts enables thiol oxidation in the reducing mitochondrial IMS. Mol. Biol. Cell 26, 195-204. doi: 10.1091/mbc.E14-10-1422

Kritsiligkou, P., Chatzi, A., Charalampous, G., Mironov, A. Jr., Grant, C. M., and Tokatlidis, K. (2017). Unconventional targeting of a thiol peroxidase to the mitochondrial intermembrane space facilitates oxidative protein folding. Cell Rep. 18, 2729-2741. doi: 10.1016/j.celrep.2017.02.053

Kurz, M., Martin, H., Rassow, J., Pfanner, N., and Ryan, M. T. (1999). Biogenesis of Tim proteins of the mitochondrial carrier import pathway: differential targeting mechanisms and crossing over with the main import pathway. Mol. Biol. Cell $10,2461-2474$

Lang, B. F., Burger, G., O’Kelly, C. J., Cedergren, R., Golding, G. B., Lemieux, C., et al. (1997). An ancestral mitochondrial DNA resembling a eubacterial genome in miniature. Nature 387, 493-497.

Lisowsky, T. (1994). ERV1 is involved in the cell-division cycle and the maintenance of mitochondrial genomes in Saccharomyces cerevisiae. Curr. Genet. 26, 15-20. doi: 10.1007/BF00326299

Longen, S., Bien, M., Bihlmaier, K., Kloeppel, C., Kauff, F., Hammermeister, M., et al. (2009). Systematic analysis of the twin cx9c protein family. J. Mol. Biol. 393, 356-368. doi: 10.1016/j.jmb.2009.08.041

Lutz, T., Neupert, W., and Herrmann, J. M. (2003). Import of small Tim proteins into the mitochondrial intermembrane space. EMBO J. 22, 4400-4408. doi: 10.1093/emboj/cdg421

Martinez-Caballero, S., Grigoriev, S. M., Herrmann, J. M., Campo, M. L., and Kinnally, K. W. (2007). Tim17p regulates the twin pore structure and voltage gating of the mitochondrial protein import complex TIM23. J. Biol. Chem. 282, 3584-3593. doi: 10.1074/jbc.M607551200

Matlack, K. E., Misselwitz, B., Plath, K., and Rapoport, T. A. (1999). $\mathrm{BiP}$ acts as a molecular ratchet during posttranslational transport of prepro-alpha factor across the ER membrane. Cell 97, 553-564. doi: 10.1016/S0092-8674(00)80767-9

Matta, S. K., Pareek, G., Bankapalli, K., Oblesha, A., and D'Silva, P. (2017). Role of Tim17 transmembrane regions in regulating the architecture of presequence translocase and mitochondrial DNA stability. Mol. Cell. Biol. 37:e00491-16. doi: 10.1128/MCB.00491-16

Meier, S., Neupert, W., and Herrmann, J. M. (2005). Conserved N-terminal negative charges in the Tim17 subunit of the TIM 23 translocase play a critical role in the import of preproteins into mitochondria. J. Biol. Chem. 280, 7777-7785. doi: 10.1074/jbc.M412158200

Mesecke, N., Terziyska, N., Kozany, C., Baumann, F., Neupert, W., Hell, K., et al. (2005). A disulfide relay system in the intermembrane space of mitochondria that mediates protein import. Cell 121, 1059-1069. doi: 10.1016/j.cell.2005.04.011
Michaelis, G., Esser, K., Tursun, B., Stohn, J. P., Hanson, S., and Pratje, E. (2005). Mitochondrial signal peptidases of yeast: the rhomboid peptidase Pcpl and its substrate cytochrome c peroxidase. Gene 354, 58-63. doi: 10.1016/j.gene.2005.04.020

Milenkovic, D., Ramming, T., Muller, J. M., Wenz, L. S., Gebert, N., SchulzeSpecking, A., et al. (2009). Identification of the signal directing Tim 9 and Tim10 into the intermembrane space of mitochondria. Mol. Biol. Cell 20, 2530-2539. doi: 10.1091/mbc.E08-11-1108

Miyata, N., Watanabe, Y., Tamura, Y., Endo, T., and Kuge, O. (2016). Phosphatidylserine transport by Ups2-Mdm35 in respiration-active mitochondria. J. Cell Biol. 214, 77-88. doi: 10.1083/jcb.201601082

Mokranjac, D., Sichting, M., Popov-Celeketic, D., Mapa, K., Gevorkyan-Airapetov, L., Zohary, K., et al. (2009). Role of Tim50 in the transfer of precursor proteins from the outer to the inner membrane of mitochondria. Mol. Biol. Cell 20, 1400-1407. doi: 10.1091/mbc.E08-09-0934

Morgan, B., and Lu, H. (2008). Oxidative folding competes with mitochondrial import of the small Tim proteins. Biochem. J. 411, 115-122. doi: 10.1042/BJ20071476

Morgenstern, M., Stiller, S. B., Lubbert, P., Peikert, C. D., Dannenmaier, S., Drepper, F., et al. (2017). Definition of a high-confidence mitochondrial proteome at quantitative scale. Cell Rep. 19, 2836-2852. doi: 10.1016/j.celrep.2017.06.014

Naoe, M., Ohwa, Y., Ishikawa, D., Ohshima, C., Nishikawa, S., Yamamoto, H., et al. (2004). Identification of Tim 40 that mediates protein sorting to the mitochondrial intermembrane space. J. Biol. Chem. 279, 47815-47821. doi: 10.1074/jbc.M410272200

Nargang, F. E., Drygas, M. E., Kwong, P. L., Nicholson, D. W., and Neupert, W. (1988). A mutant of Neurospora crassa deficient in cytochrome c heme lyase activity cannot import cytochrome c into mitochondria. J. Biol. Chem. 263, 9388-9394

Neal, S. E., Dabir, D. V., Wijaya, J., Boon, C., and Koehler, C. M. (2017). Osm1 facilitates the transfer of electrons from Ervl to fumarate in the redox-regulated import pathway in the mitochondrial intermembrane space. Mol. Biol. Cell 28, 2773-2785. doi: 10.1091/mbc.E16$10-0712$

Neupert, W., and Brunner, M. (2002). The protein import motor of mitochondria. Nat. Rev. Mol. Cell Biol. 3, 555-565. doi: 10.1038/nrm878

Nicholson, D. W., and Neupert, W. (1989). Import of cytochrome c into mitochondria: reduction of heme, mediated by $\mathrm{NADH}$ and flavin nucleotides, is obligatory for its covalent linkage to apocytochrome c. Proc. Natl. Acad. Sci. U.S.A. 86, 4340-4344. doi: 10.1073/pnas.86. 12.4340

Ohsato, T., Ishihara, N., Muta, T., Umeda, S., Ikeda, S., Mihara, K., et al. (2002). Mammalian mitochondrial endonuclease G. Digestion of R-loops and localization in intermembrane space. Eur. J. Biochem. 269, 5765-5770. doi: 10.1046/j.1432-1033.2002.03238.x

Okamoto, K., Brinker, A., Paschen, S. A., Moarefi, I., Hayer-Hartl, M., Neupert, W., et al. (2002). The protein import motor of mitochondria: a targeted molecular ratchet driving unfolding and translocation. EMBO J. 21, 3659-3671. doi: $10.1093 / \mathrm{emboj} / \mathrm{cdf3} 38$

Peleh, V., Cordat, E., and Herrmann, J. M. (2016). Mia40 is a trans-site receptor that drives protein import into the mitochondrial intermembrane space by hydrophobic substrate binding. Elife 5:e16177. doi: 10.7554/eLife.16177

Peleh, V., Zannini, F., Backes, S., Rouhier, N., and Herrmann, J. M. (2017). Erv1 of Arabidopsis thaliana can directly oxidize mitochondrial intermembrane space proteins in the absence of redox-active Mia40. BMC Biol. 15:106. doi: 10.1186/s12915-017-0445-8

Ramesh, A., Peleh, V., Martinez-Caballero, S., Wollweber, F., Sommer, F., van der Laan, M., et al. (2016). A disulfide bond in the TIM23 complex is crucial for voltage gating and mitochondrial protein import. J. Cell Biol. 214, 417-431. doi: 10.1083/jcb.2016 02074

Rissler, M., Wiedemann, N., Pfannschmidt, S., Gabriel, K., Guiard, B., Pfanner, N., et al. (2005). The essential mitochondrial protein Erv1 cooperates with Mia40 in biogenesis of intermembrane space proteins. J. Mol. Biol. 353, 485-492. doi: 10.1016/j.jmb.2005.08.051

Schendzielorz, A. B., Schulz, C., Lytovchenko, O., Clancy, A., Guiard, B., Ieva, R., et al. (2017). Two distinct membrane potential-dependent steps drive mitochondrial matrix protein translocation. J. Cell Biol. 216, 83-92. doi: $10.1083 /$ jcb.201607066 
Schulz, C., Lytovchenko, O., Melin, J., Chacinska, A., Guiard, B., Neumann, P., et al. (2011). Tim50's presequence receptor domain is essential for signal driven transport across the TIM23 complex. J. Cell Biol. 195, 643-656. doi: $10.1083 /$ jcb. 201105098

Shiota, T., Imai, K., Qiu, J., Hewitt, V. L., Tan, K., Shen, H. H., et al. (2015), Molecular architecture of the active mitochondrial protein gate. Science 349, 1544-1548. doi: 10.1126/science.aac6428

Sickmann, A., Reinders, J., Wagner, Y., Joppich, C., Zahedi, R., Meyer, H. E., et al. (2003). The proteome of Saccharomyces cerevisiae mitochondria. Proc. Natl. Acad. Sci. U.S.A. 100, 13207-13212. doi: 10.1073/pnas.2135385100

Sideris, D. P., Petrakis, N., Katrakili, N., Mikropoulou, D., Gallo, A., CiofiBaffoni, S., et al. (2009). A novel intermembrane space-targeting signal docks cysteines onto Mia40 during mitochondrial oxidative folding. J. Cell Biol. 187, 1007-1022. doi: $10.1083 /$ jcb. 200905134

Sideris, D. P., and Tokatlidis, K. (2007). Oxidative folding of small Tims is mediated by site-specific docking onto Mia40 in the mitochondrial intermembrane space. Mol. Microbiol. 65, 1360-1373. doi: 10.1111/j.1365-2958.2007.05880.x

Sirrenberg, C., Bauer, M. F., Guiard, B., Neupert, W., and Brunner, M. (1996). Import of carrier proteins into the mitochondrial inner membrane mediated by Tim22. Nature 384, 582-585. doi: 10.1038/384582a0

Slutsky-Leiderman, O., Marom, M., Iosefson, O., Levy, R., Maoz, S., and Azem, A. (2007). The interplay between components of the mitochondrial protein translocation motor studied using purified components. J. Biol. Chem. 282, 33935-33942. doi: 10.1074/jbc.M704435200

Sztolsztener, M. E., Brewinska, A., Guiard, B., and Chacinska, A. (2013). Disulfide bond formation: sulfhydryl oxidase ALR controls mitochondrial biogenesis of human MIA40. Traffic 14, 309-320. doi: 10.1111/tra.12030

Terziyska, N., Lutz, T., Kozany, C., Mokranjac, D., Mesecke, N., Neupert, W., et al. (2005). Mia40, a novel factor for protein import into the intermembrane space of mitochondria is able to bind metal ions. FEBS Lett. 579, 179-184. doi: 10.1016/j.febslet.2004.11.072

Tienson, H. L., Dabir, D. V., Neal, S. E., Loo, R., Hasson, S. A., Boontheung, P., et al. (2009). Reconstitution of the mia40-erv1 oxidative folding pathway for the small tim proteins. Mol. Biol. Cell 20, 3481-3490. doi: 10.1091/mbc.E08-10-1062

Ting, S. Y., Yan, N. L., Schilke, B. A., and Craig, E. A. (2017). Dual interaction of scaffold protein Tim44 of mitochondrial import motor with channel-forming translocase subunit Tim23. Elife 6:e23609. doi: 10.7554/eLife.23609

Truscott, K. N., Kovermann, P., Geissler, A., Merlin, A., Meijer, M., Driessen, A. J., et al. (2001). A presequence- and voltage-sensitive channel of the mitochondrial preprotein translocase formed by Tim23. Nat. Struct. Biol. 8, 1074-1082. doi: $10.1038 / \mathrm{nsb} 726$

van der Laan, M., Meinecke, M., Dudek, J., Hutu, D. P., Lind, M., Perschil, I., et al. (2007). Motor-free mitochondrial presequence translocase drives membrane integration of preproteins. Nat. Cell Biol. 9, 1152-1159. doi: 10.1038/ncb1635
Vögtle, F. N., Burkhart, J. M., Rao, S., Gerbeth, C., Hinrichs, J., Martinou, J. C., et al. (2012). Intermembrane space proteome of yeast mitochondria. Mol. Cell. Proteomics 11, 1840-1852. doi: 10.1074/mcp.M112.0 21105

Vögtle, F. N., Wortelkamp, S., Zahedi, R. P., Becker, D., Leidhold, C., Gevaert, K., et al. (2009). Global analysis of the mitochondrial N-proteome identifies a processing peptidase critical for protein stability. Cell 139, 428-439. doi: 10.1016/j.cell.2009.07.045

Voisine, C., Craig, E. A., Zufall, N., von Ahsen, O., Pfanner, N., and Voos, W. (1999). The protein import motor of mitochondria: unfolding and trapping of preproteins are distinct and separable functions of matrix Hsp70. Cell 97, 565-574. doi: 10.1016/S0092-8674(00)80768-0

von der Malsburg, K., Muller, J. M., Bohnert, M., Oeljeklaus, S., Kwiatkowska, P., Becker, T., et al. (2011). Dual role of mitofilin in mitochondrial membrane organization and protein biogenesis. Dev. Cell 21, 694-707. doi: 10.1016/j.devcel.2011.08.026

Wang, C., and Youle, R. J. (2009). The role of mitochondria in apoptosis. Annu. Rev. Genet. 43, 95-118. doi: 10.1146/annurev-genet-102108-1 34850

Weckbecker, D., Longen, S., Riemer, J., and Herrmann, J. M. (2012). Atp23 biogenesis reveals a chaperone-like folding activity of Mia40 in the IMS of mitochondria. EMBO J. 31, 4348-4358. doi: 10.1038/emboj.2012.263

Wrobel, L., Trojanowska, A., Sztolsztener, M. E., and Chacinska, A. (2013). Mitochondrial protein import: mia40 facilitates Tim22 translocation into the inner membrane of mitochondria. Mol. Biol. Cell 24, 543-554. doi: 10.1091/mbc.E12-09-0649

Yamano, K., Kuroyanagi-Hasegawa, M., Esaki, M., Yokota, M., and Endo, T. (2008). Step-size analyses of the mitochondrial Hsp70 import motor reveal the Brownian ratchet in operation. J. Biol. Chem. 283, 27325-27332. doi: 10.1074/jbc.M805249200

Young, J. C., Hoogenraad, N. J., and Hartl, F. U. (2003). Molecular chaperones Hsp90 and Hsp70 deliver preproteins to the mitochondrial import receptor Tom70. Cell 112, 41-50. doi: 10.1016/S0092-8674(02)01250-3

Zarsky, V., and Dolezal, P. (2016). Evolution of the Tim17 protein family. Biol. Direct 11:54. doi: 10.1186/s13062-016-0157-y

Conflict of Interest Statement: The authors declare that the research was conducted in the absence of any commercial or financial relationships that could be construed as a potential conflict of interest.

Copyright (C) 2017 Backes and Herrmann. This is an open-access article distributed under the terms of the Creative Commons Attribution License (CC BY). The use, distribution or reproduction in other forums is permitted, provided the original author(s) or licensor are credited and that the original publication in this journal is cited, in accordance with accepted academic practice. No use, distribution or reproduction is permitted which does not comply with these terms. 